Dr. Leopoldo Tillería Aqueveque

Universidad Tecnológica de Chile INACAP

@ leopoldo.tilleria@inacapmail.cl (iD 0000-0001-5630-7552
- Recibido / Received 18 de septiembre de 2020

- Aceptado / Acepted 5 de octubre de 2020

- Páginas / Pages De la 335 a la 347

- ISSN: 1885-365X

\title{
Ge-stell, simulacro y matrix: Dispositivos de sentido en Heidegger, Baudrillard y Holzapfel
}

\author{
Ge-stell, simulacrum and matrix: Devices of \\ sense in Heidegger, Baudrillard and Holzapfel
}

\section{RESUMEN:}

Se desarrolla una breve exégesis de los planteamientos de Heidegger, Baudrillard y Holzapfel, que describirían una sociedad posmoderna al borde del vacío o, si se prefiere, al límite del sin-sentido. Dichos marcos ontológicos -el Ge-stell, el simulacro y la matrix, respectivamente- remiten no solo a un sobre-poblamiento de datos, sino particularmente a un despliegue, por decirlo así, irrefrenable de dispositivos tecnológicos. Guardando las distancias teóricas del caso, estos tres pensadores, cada uno a su manera, parecen describir en qué consistiría este Leviatán posmoderno, formado por aplicaciones, interfaces, el $6 G$ y toda gama de hiperconexiones de la que es capaz hoy día la tecnología. Se tiene en cuenta la conjetura de Malabou, para quien, a propósito de su recepción del Ge-stell heideggeriano, los objetos tecnológicos nunca se presentan como objetos vacíos, sino que siempre suponen una cierta mutación ontológica. Tal transformación, desde luego, es la de nosotros mismos. Una visión futurista perfectamente pudiera mostrar a una tribu de replicantes, armados - es casi seguro- de una tecnología hoy inimaginable, pero, sin embargo, abocados a esa tarea casi mística de dotación de sentido.

\section{PALABRAS CLAVE:}

Ge-stell; Matrix; Sentido; Simulacro; Tecnología; Vacío.

\section{ABSTRACT:}

A brief exegesis of the approaches of Heidegger, Baudrillard and Holzapfel is developed, which would describe a postmodern society on the edge of emptiness or, if you prefer, on the edge of nonsense. Said ontological frames -the Ge-stell, the simulacrum and the matrix, respectively - refer not only to an overpopulation of data, but particularly to an unstoppable deployment, so to speak, of technological devices. Keeping the theoretical distances of the case, these three thinkers, each in their own way, seem to describe what this postmodern Leviathan would consist of, made up of applications, interfaces, $6 \mathrm{G}$ and all the range of hyperconnections that technology is capable of today. Malabou's conjecture is taken into account, for whom, regarding his reception of the Heideggerian Ge-stell, technological objects are never presented as empty objects, but always suppose a certain ontological mutation. Such transformation, of 
course, is that of ourselves. A futuristic vision could perfectly show a tribe of replicants, armed -it is almost certain - with a technology unimaginable today, but, nevertheless, engaged in this almost mystical task of providing sense.

KEY WORDS:

Ge-stell; Matrix; Sense; Simulacrum; Technology; Emptiness.

\section{Introducción}

En el Leviatán -su obra más reconocida y cuyos efectos histórico-políticos podrían ser, perfectamente, equiparados a los del Príncipe de Maquiavelo-, Thomas Hobbes (1980) define a esta especie de bestia de siete cabezas como un gran animal artificial creado por el hombre, que adquiere su fisonomía definitiva en esa extraña mezcla de vida y artificio que invoca el pensador inglés en la idea del Estado moderno. Pues bien, en cierto modo considero que la información del siglo xxl, a la que se ha dado en llamar hiperinformación (Han, 2014), se impone hoy con una fuerza descomunal y una inevitabilidad tal, que pareciera adquirir no solo la forma, sino también la función del Leviatán hobbesiano. La sociedad posmoderna parece sobre-poblar de datos cada uno de los espacios en que ella misma se constituye: los íntimos, los domésticos, los sociales y, para llamar así a todos aquellos fenómenos acelerados por el uso de las tecnologías, los globales. Dicho sobre-poblamiento equivale a la intención de diseñar un modelo de descripción de la realidad, cuyo espesor esté determinado por la generación, intercambio, transferencia, desecho y renovación de información en sus múltiples y prácticamente infinitos soportes y plataformas. En tal medida, la idea de horror vacui adquiere un peso decisivo. Si la posmodernidad ha acelerado este «repoblamiento» de datos de nuestras esferas pública y privada, lo ha hecho a costa de una realidad que ha ido mostrando señales cada vez más fuertes de «despoblamiento» de sentido. En efecto, la era de la técnica, anunciada no solo por Heidegger, sino también por pensadores como Dessauer, Mumford, Sloterdijk e incluso el mismo Ortega, se ha movido en una relación casi directa con nuestra pérdida de sentido. Esta suerte de angustia frente a la ausencia de sentido -y esta sería nuestra principal conjetura-, ha determinado algo parecido a un «giro hiperinformativo», que se expresaría fundamentalmente en un culto a la información.

Nos enfrentamos, nolens volens, a la era del dispositivo de datos. La hiperinformación parece «purgar» la ausencia de sentido y apostar a llenar este vacío, mediante justamente un sinfín de fuentes de sentido. Al respecto, resultan vigentes para describir este fenómeno las críticas de diferentes autores que han tratado de explicar no solo los fundamentos, sino los alcances de este Leviatán del siglo xxI. Entre estos, Heidegger, (1953, 1955), Vattimo (1974, $1981,1983)$, Baudrillard (1978, 1980, 1983, 1990), Holzapfel $(2005,2012,2018,2020)$ y Puig $(2015,2016)$. Tres de ellos me parecen indispensables: Heidegger y su idea de Ge-stell; Baudrillard y su concepto de hiperrealidad; y Holzapfel, a través de la pregunta por el sentido. La argumentación va más bien por el lado de reconocer - siguiendo en esto a Heidegger - a la sociedad actual como la era del Ge-stell. Si lo que se desoculta por medio del Ge-stell es precisamente nuestro modo de ser técnico, o tecnológico, entonces caemos en la cuenta de que en el centro de este dispositivo se halla sobre todo la información hiperexpuesta. Si pudiera decirse así: el big data. En un plano ontológico diferente, Baudrillard ha descrito la era actual como la de la hiperrealidad, el punto en que la idea de simulacro ha rebasado toda posibili- 
dad de distingo entre verdad y simulación, entre realidad y no realidad. La hiperrealidad es, pues, una respuesta desde el hastío. A partir del exceso de información, tiende a promover otras performances y posibilidades de determinación de la posverdad. Por su parte, el filósofo chileno Cristóbal Holzapfel realizará una interpelación esencial a los tiempos que corren. ¿En qué medida la pregunta por el sentido es en realidad la última posibilidad de contrarrestar el vacío por antonomasia que parece ser la nada? Toda la antropología filosófica de Holzapfel, gira en torno a ese torbellino ontológico en que la nada nos acosa y abruma a partir de un sin-sentido fundamental.

En todo caso, la presencia de estos autores, aparentemente tan diferentes en «programas» y estilos, obedece no solo al rótulo general de filósofos del sentido, cuestión que en principio tendría que ver con el título del manuscrito, sino especialmente con su preocupación, dicho de manera radical, por el problema del fundamento. De esta laya, Heidegger, Holzapfel y Baudrillard, compartirían en sus filosofías una misma dirección ontológica, lo que Navarro Cordón (1966) denomina Fundamentalontologie.

El escrito (una breve exégesis respecto de los autores indicados) se organiza en tres secciones: primero, se discute la posibilidad de entender la hiperinformación como una dimensión del Ge-stell heideggeriano, provista de credenciales primeramente ontológicas. Enseguida, se pone en liza la idea de Baudrillard de hiperrealidad, dentro de lo que pudiéramos llamar una cultura del simulacro. Sobre el final, se describen las concomitancias en Holzapfel entre los conceptos de matrix y de fuentes dispensadoras de sentido.

\section{El Ge-stell como mutación onto-tecnológica}

En La pregunta por la técnica (1953), una de las conferencias clave después de su rectorado en la Universidad de Friburgo, Heidegger define la esencia de la técnica moderna como Ge-stell, es decir, como aquel modo de ser en el mundo en que la técnica «envuelve» al ser humano en todos los planos de su existencia. Dice Heidegger (1983): «Lo dis-puesto [el Ge-stell] es lo que reúne a aquel poner, que pone al hombre a desocultar lo real en el modo del establecer como constante» (p. 92). Se trata, diríamos hoy, de la imposición del mundo tecnológico.

He utilizado en el mismo pasaje las nociones de técnica y tecnología, a sabiendas de que, si bien es cierto no son sinónimos, forman un mismo núcleo semántico a la hora de empeñarse en comprender las complejidades de la era de la hiperinformación. Creo necesario, no obstante, esclarecer su recepción. Evidentemente, entenderé técnica, como quien dice, al modo heideggeriano, es decir, no aun en su consideración esencial -dictum completo de La pregunta por la técnica», sino en su determinación instrumental o antropológica. Visto así, la técnica sería una ejecución, «un medio y un hacer del hombre» (Heidegger, 1983: 72). Sin embargo, esta concepción «utilitaria» no puede sino entenderse en conexión con la idea fundamental que desarrolla el filósofo en su texto sobre la técnica, es decir, comprendiéndola como una fuerza no controlable y manipulable enteramente por el hombre (Linares, 2003: 17). En esta misma línea -y lo traigo a colación solo para dar mayor consistencia a la referencia-, se halla la definición de técnica de Quintanilla (2005), para quien esta «es una clase de realizaciones técnicas equivalentes respecto al tipo de acciones, a su sistematización, a 
las propiedades de los objetos sobre los que se ejercen y a los resultados que se obtienen» (p. 47). En cambio, la tecnología será entendida como la organización sistemática de la técnica. Dicho llanamente, como un medio o instrumento que sirve para algo definido por sus creadores (López Araiza Bravo, 2012: 117). Sintetizando ambas definiciones, podría decirse que la tecnología es la instrumentación de la acción técnica. En razón de esto, la relación ontológica técnica/tecnología correspondería a la de acción/objeto. Ahora, la nomenclatura global de este escrito las abordará, en general, como conceptos equivalentes. Conviene, al respecto, considerar la idea de López Devesa: «Si entendemos la tecnología dentro del complejo entramado social en el que surge, podremos comprender que técnica y tecnología no se diferencian esencialmente» (López Devesa, 2001: 199).

Volviendo a Heidegger, el desocultar provocante del que habla el filósofo de Friburgo, ocurre desde y en la existencia misma de los seres humanos. Nos vemos obligados a convivir en todo momento con las determinaciones técnico-tecnológicas del mundo global. A esta mentalidad de la era de la técnica, rebasada de la idea de lo técnico, Heidegger la llamará Ge-stell, y a los objetos propios de esta maniobra tecnológica, es decir, a la naturaleza volcada en y absorbida por la técnica y la tecnología, constante. De este modo, el panorama que nos ofrece Heidegger es el de un mundo permeado por todas partes por la tecnología, transido de artefactos, aplicaciones, dispositivos e información, únicamente conducente al gerenciamiento técnico de los seres humanos (Feenberg, 2005). Al respecto, y en una brevísima digresión, consignemos que, para efectos de diferenciar los términos dato, información y conocimiento, es decir, en una especificación ontológica de la idea de hiperinformación, seguiremos la postura de Boisot y Canals (2004), para quienes los datos son estímulos sensoriales y diferenciables que captamos mediante nuestros sentidos (Guillén et al., 2015: 156). Del mismo modo, entenderemos información como el resultado de procesar e interpretar los datos de manera que nos aporten significado (Guillén et al., 2015: 156). Es decir, sería algo así como la semántica de los datos. Por último, y dentro de las múltiples definiciones posibles de conocimiento, digamos que equivale a la comprensión de la información que nos hemos apropiado e interiorizado, esto es, al resultado de procesar la información y de hallar ciertos patrones invariantes que generan un corpus de juicios acerca del mundo (Guillén et al., 2015: 157).

Ahora bien, y como indica Borges Duarte (1993), la esencia de la técnica

(...) consiste en la forma característica que la realidad gana en el mundo en el que se da la consumación nihilista de la historia de Occidente, es decir, en la figura-esbozo-proyección de una imagen del mundo ideológica, tecnológicamente trazada y programada (Borges Duarte (1993), cit. en Salomón Amaya, 2015: 61)

Sin embargo -y aquí probablemente adquiere más sentido la idea de horror al vacío-, este desocultar se presenta al mismo tiempo, dice Heidegger (1983), bajo la forma de un peligro: «La amenaza no le viene al hombre principalmente de que las máquinas y aparatos de la técnica puedan actuar quizás de modo mortífero. La más peculiar amenaza se ha introducido ya en la esencia del hombre» (p. 97). El mayor peligro en la era de la técnica se muestra, paradójicamente, de la mano de la propia esencia de la técnica. Ahora bien, este Ge-stell no resulta un simple dispositivo tecnológico, ni mucho menos. El Ge-stell resulta ser una cierta forma de disponer y comprender la tecnología, de tal manera que podamos enfocarnos - voluntariamente o no- justamente en aquel, y no en otro, rendimiento maquinal. Observa Luna Alcoba: 
El dispositivo lo que hace es situar a la máquina en dis-posición de hacer cosas [...] es un sistema que está preparado para generar determinadas respuestas ante determinados inputs. Esa relación entre entradas y salidas halla su fundamento en la dis-posición. Pero, a su vez, el dispositivo también nos dis-pone a nosotros a establecer una y solo una relación concreta con él [...] De aquí la disposición sea un dispersor de enunciados, de trayectorias, de comportamientos, impulsados, empujados por el dispositivo que los modifica, los altera (Luna Alcoba, 2013: 60).

El Ge-stell se constituye en realidad como un sistema tecnológico, que, a su vez, nos determina a mantener un determinado tipo de relación ontológica con él. Dicho en corto: se trata de un circuito, y todo circuito, mediante sus dispositivos, transmite, comprime, genera o conmuta datos y procesos, es decir, transforma información. El Ge-stell nos informa acerca de este nuevo estado del arte tecnológico, haciéndonos parte fundamental de él. De ahí que la interpretación de Malabou (2011) sea tan lúcida, en el sentido de que concibe al Ge-stell como un virtual «transformador», cuya principal tarea es precisamente la de la convertibilidad ontológica: «El Gestell es un ser que permite que su esencia le sea dada. [...] Si esta sustituibilidad atestigua una metamorfosis y una migración de la esencia, al mismo tiempo revela una metamorfosis y migración de los propios seres" (Malabou, 2011: 165). De tal manera que la mentada esencia de la tecnología, que corresponde a una de las definiciones ortodoxas del Ge-stell, se transforma, en la misma manipulación de los seres tecnológicos, en la propia mutabilidad ontológica de cada uno de ellos. Subraya Malabou:

Los seres tecnológicos ya no son los seres de la existencialidad. Los seres se han vuelto porosos, esencialmente porosos, y esto permite que la verdad se transmita a través de ellos sin que esto los presente (vorhanden). Es por eso que un ser tecnológico nunca es simplemente un programa. Siempre es al mismo tiempo una promesa, que abre automáticamente la puerta a lo esencial. Lo que dice el Gestell es precisamente este punto de indistinción entre el programa - automatismo - y la promesa, lo esencial (Malabou (2011: 166-167).

Como lo expone Catherine Malabou, cuando sostengo un objeto tecnológico en mis manos, nunca lo sostengo solo, sino esencialmente como resultado de una mutación ontológica.

\section{Simulacro, hiperrealidad, alucinación}

Jean Baudrillard desarrolla en varios de sus textos uno de los conceptos clave de la posmodernidad: el de simulacro. Este ya no implica, dice el francés, como desde los griegos hasta esta parte, la idea de mímesis, en cualesquiera de sus variantes o apropiaciones. Simulacro implica una nueva forma de interpretar la realidad posmoderna: literalmente, una nueva realidad. Como advierte Vaskes Santches: «Baudrillard elabora una visión de la realidad contemporánea como simulación total que tiende a subvertir la representación, a destruir toda su lógica referencial y significativa, haciendo desaparecer la realidad y enmascarar al mismo tiempo su desaparición» (Vaskes Santches, 2008: 202). En El intercambio simbólico y la muerte (1980), Baudrillard describirá los tres tipos de simulacros que han estado presentes prácticamente en toda la historia de la humanidad. En particular, quiero detenerme en el primero, que corresponde exactamente a la idea de simulación, es decir, «el esquema dominante de la fase actual regida por el código» (Baudrillard, 1980: 59). La simulación constituye 
el estadio avanzado del simulacro, cuyas fases preliminares han sido descritas por el filósofo galo como las de la falsificación y la de la producción. La simulación es, pues, un modelo construido de una vez mediante la respuesta activa del sujeto. Sostiene Baudrillard: «jHe aquí la llegada de la gran Cultura de la comunicación táctil, bajo el signo del espacio tecno-lumino-cinético y del teatro total espaciodinámico!» (1980: 84). En consecuencia, el simulacro de la fase posmoderna no es sino la simulación de una nueva realidad, la de lo hiperreal:

Lo hiperreal representa una fase mucho más avanzada, en la medida en que incluso esta contradicción de lo real y lo imaginario, queda en él borrada. La irrealidad no es en él la del sueño o del fantasma, de un más allá o de un más acá, es la de la alucinante semejanza de lo real consigo mismo (Baudrillard, 1980, p. 85).

La crítica baudrillardiana resulta ser, en primer lugar, una crítica ontológica. Mediante la idea de simulación, lo que se asevera es que la propia realidad ha desaparecido, y que es tarea de cada uno de nosotros «expiar» esa desaparición, por medio no de la «producción» de simulaciones (esto es imposible por la propia naturaleza de lo que se simula), sino a través de la aceptación de la idea de ilusión radical. Tal idea es el principio de disolución de la realidad. Señala Vaskes Santches: «Esta apreciación es especialmente importante para el mundo actual, en cuyo contexto de las tecnologías digitales e informáticas cada vez más sofisticadas, la relación entre verdad y ficción es mucho más compleja» (2008: 204). Más aún, lo esencial de la hiperrealidad es justamente la inversión del movimiento del simulacro: los simuladores, dirá Baudrillard, tienen la tarea de hacer que la misma realidad coincida con sus modelos y algoritmos de simulación. Algo radical parece haber ocurrido en el orden de la realidad, tan inesperado como su propia desaparición. Esta especie de vacuidad, si seguimos a Baudrillard, la llena el ser humano (la familia, los medios, los gobiernos, las agencias, Internet) precisamente por medio de un exceso de información:

Los medios tienen un lugar especial en la implosión del significado. En particular, crean una presión de información excesiva. Esto lleva a la desestructuración de lo social, pero al mismo tiempo, los medios alientan una sensación de seguridad. Incluso cuando presentan violencia o desastres, el hecho de no "estar allí» mientras se observa, refuerza la seguridad. A través de los medios, nunca alcanzamos el evento real, sino solo su suplemento informativo, que está abierto a una interminable interpretación (Leyton, 2018).

Se trata de una realidad simplemente alucinatoria. Por lo mismo, la hiperrealidad muestra un mundo virtual de alta definición, de perfección absoluta, que configura lo real como totalidad, eliminando la ilusión y cualquier alternativa imaginaria (Vakes Santches, 2008). Dado que ontológicamente no hay diferencia entre ilusión y realidad, la sociedad global se hallaría en una suerte de tabula rasa para generar, tal como lo veíamos con la idea del Ge-stell, todo tipo de diseño, plataforma o performance que la pueda definir. La hiperrealidad equivale al propio dispositivo de contrarresto del terror al vacío causado por el desencanto de la producción. A propósito de la incrustación de lo hiperreal en el mundo del arte, dirá Baudrillard

El hiper-realismo es el colmo del arte y el colmo de lo real por intercambio respectivo, al nivel del simulacro, de los privilegios y de los prejuicios que los fundan. Lo hiper-real no está más allá de la representación (cf. J.-F. Lyotard, L'Art Vivant, número sobre el hiper-realismo) sino porque está enteramente en la simulación. (1980: 87) 
Todo queda cubierto por los artificios de lo simulado: desde los medios (reality shows) hasta la vida familiar (dispositivos del Internet de las cosas y softwares para el teletrabajo en condiciones de pandemia), desde los géneros del arte (el trompe-l'oeil y la pintura hiperrealista de Bell, Degraaf o de Campos) hasta la investigación aeroespacial (El Modelo Virtual del Espacio Aéreo y el proyecto de Simulación VAMS de la NASA), desde las ciencias médicas (softwares de telesimulación y teledebriefing) hasta el erotismo (la simulación masturbatoria mediante todo tipo de «implementos» físicos y químicos). Leemos en De la seducción:

Visto de muy cerca, se ve lo que no se había visto nunca - su sexo, usted no lo ha visto nunca funcionar, ni tan de cerca, ni tampoco en general, afortunadamente para usted. Todo eso es demasiado real, demasiado cercano para ser verdad. Y eso es lo fascinante, el exceso de realidad, la híperrealidad de la cosa. El único fantasma en juego en el porno, si es que hay uno, no es el del sexo, sino el de lo real, y su absorción, absorción en otra cosa distinta de lo real, en lo hiperreal (Baudrillard, 2000: 33).

El fin de los relatos, advertido por Lyotard a fines de los setenta, ha requerido que la era del Internet pueble de simulaciones prácticamente todas las facetas de nuestra vida. De esta forma, se asiste a una perfecta indistinción de sujeto y objeto. Incluso, en la propia realidad del objeto, su simulación toma una preeminencia solo transitoria, pues una nueva simulación (la versión 2.0, 3.0 o la que corresponda al modelo en cuestión) hará las veces de actualización de ese instante de hiperrealidad. Diríamos, parafraseando a McLuhan, que el medio no es solo el mensaje, sino literalmente la propia realidad. Una de las mayores masacres del siglo XXI, perpetrada por Brenton Tarrant en marzo de 2019 en las mezquitas de Nueva Zelanda, fue «transmitida» en tiempo real por su autor desde la cámara GoPro adosada a su casco, mediante Facebook Live. Mientras iban cayendo sus víctimas, los millones de usuarios de redes sociales, que veían en directo el desplazamiento del francotirador, virtualmente era parte de una de las varias jugadas posibles de Free Fire o Fortnite. La realidad, pues, simulaba in toto cada una de las modalidades tecnológicas de un juego de combate en línea, transformando - muy cuestionablemente - la masacre en trending topic, y llenando Twitter de mensajes y cápsulas del video que los usuarios «afortunadamente» habían logrado guardar. No olvidemos, por otro lado, que Facebook puede sugerirnos a determinadas personas para etiquetar en una publicación solo con detectar su rostro en una foto, en un claro ejemplo de los alcances que puede tener este tipo de tecnología (Ramos, 2019).

\section{La matrix de Holzapfel}

La teoría de Cristóbal Holzapfel encubre una crucial y acaso inacabada pregunta por el sentido. Subraya el filósofo chileno en $A$ la búsqueda del sentido:

Mas, está claro que vivir en esa cercanía a la que llamamos «trasfondo", en el que puede comparecer tanto el sentido como el sin-sentido, resulta desazonador para el común de las personas. Si nos representamos esto como una escalera de Jacob invertida, vale decir, no una que sube al cielo y por donde suben y bajan ángeles, sino una que desciende a los abismos, al filósofo le corresponde bajar con tesón y resolución hasta las profundidades, mas puede sucederle a quienes hacen este intento que ya en el peldaño número 30 comiencen a sentir un vértigo irresistible (Holzapfel, 2005: 17). 
Tal vértigo, nos obliga a recurrir a lo que el autor llamará fuentes dispensadoras de sentido: amor, amistad, poder, saber, juego, ciencia, técnica, política, etc. Desde luego, este largo etcétera incluye también a la tecnología. Para ordenar el argumento, digamos que en Holzapfel se trata eminentemente de nuestra relación con el mundo, donde el énfasis parece estar puesto en la idea de «relación». En efecto, dado que la posmodernidad bien podría definirse como la era de la crisis de la representación, caemos en la cuenta de que la propia teoría representacional, dada su ostensiva vinculación con la cuestión de los límites, pudiera arrojar también sentidos de la nada (Holzapfel, 2018: 83). El vértigo, la nada existencial u ontológica, equivale, de un modo u otro, a la pérdida del sentido. Escribe Holzapfel:

La nada determina al hombre en su referencia al pasado que ya no es en cuanto recuerda, y en cuanto imagina y proyecta se orienta a un futuro que todavía no es [...] Incluso la tecnología, la economía, la política, y otras ocupaciones humanas, se refieren también de distinta forma a lo que ya no es o que todavía no es (Holzapfel, 2005: 230-231).

Paradójicamente este sin-sentido, piensa Holzapfel, está a un tris del propio sentido. En otras palabras, enfrentamos esta posibilidad del sin-sentido mediante una serie de fuentes dispensadoras, las que nos pondrían en una situación de aparente dotación definitiva de sentido. Sin embargo, esta convicción nunca queda «a firme», siempre nos deja al borde del sin-sentido. El único modo de enfrentar esta verdadera espada de Damocles es «sostenerse en el trasfondo", en nuestra fuente última de sentido. De hecho, si pudiera hablarse en cierto modo de estrategias de sentido, diríamos, siguiendo el comentario de Villarino, que el sentido va encadenado:

\footnotetext{
El matrimonio, por ejemplo, es una fuente persistente de sentido solo si está relacionada con las fuentes referenciales del amor, el juego y la amistad. La vida ética quizá sólo tenga sentido si está nutrida por la voz de la conciencia [...] Holzapfel no se plantea solo la nuda dialéctica realidad-irrealidad, establece una gradación de realidad donde la inferior de algún modo adeuda su propio ser a la superior; para ser depende en cierto modo de su vínculo con ella y suscita inexorablemente la pregunta por la realidad del trasfondo del que penden todos los sentidos (Villarino, 2008: 74).
}

Para Holzapfel, el sentido se expresa principalmente en tres ámbitos: el semántico, el existencial y el metafísico (2005: 14). Estos aspectos forman un ir y venir del sentido por medio de la articulación de un cierto logos, o, como más arriba pergeñábamos, de la representación del mundo. De alguna manera, y Holzapfel lo detalla a cabalidad en Nada (2018), este logos del sentido es precisamente el que determina lo que los entes son y cómo se comportan: «En cierto modo, ahí está la matrix de todo. Probablemente, la tarea a futuro de las ciencias naturales sea el ingreso en esa matrix» (Holzapfel, 2018: 59). Dicha matrix, por tanto, parece hacer las veces de un escudo de información, para no caer en el vacío del «otro» lado del trasfondo, justamente el punto de no retorno del sinsentido. Como sea, no debiera sorprender esta alusión a la idea de la matrix como diseño de la realidad. Ya Bostrom (2003) ha sugerido que no es inadmisible que en la historia del universo la tecnología se desarrolle de tal forma, que se puedan crear simulaciones computarizadas de mundos enteros (Chalmers, 2007). Si esto es así, puede haber muchos más seres que están en una matrix que seres que no lo 
están, de lo que se puede inferir, incluso, que es más probable que estemos en una a que no lo estemos (Chalmers, 2007: 212).

Ahora bien, desde una perspectiva cosmológica, Holzapfel enfatizará las consideraciones de la astrofísica moderna respecto del lugar que ocuparíamos como planeta en la idea general del universo. Por cierto, si se llegara a corroborar definitivamente la concepción del «multiverso", esto «significaría que con todo aquello seríamos nada más que una gota en el océano» (Holzapfel, 2020). Hay un cálculo que refleja en toda su expresión el verdadero horror vacui que supone, cambiando los términos, ser esa gota de leche en la Vía Láctea. Holzapfel se remite, una vez más, al tiempo. La comparación en cuestión establece que, si se considerara el tiempo transcurrido desde la primera civilización humana en unos 25.000 años, en relación con el tiempo en que teóricamente el sol completa una sola vuelta alrededor del eje de la Vía Láctea, unos 225 millones de años, seríamos, entonces, parece decir perplejo Holzapfel (2020), un 0,0001\% de ese recorrido. Quiérase o no, esta perpetua danza en torno al astro rey nos devuelve a la pregunta original por el sentido. Dicha pregunta no puede sino inquirir al mismo tiempo por la nada: «Dado que, si el ser tuviera límites absolutos tanto hacia lo mínimo, el micro-ser, como hacia lo máximo, el macro-ser, ¿qué hay antes o después, más acá o más allá de esos límites? ¿El no ser? ¿La nada?» (Holzapfel, 2020). Seríamos, pues, una "gota de leche» en la infinita esfera del universo y el ser. Frente a este vacío cosmológico y ontológico, Holzapfel postulará, con apoyo en Weischedel (1971/72), la noción de fuentes dispensadoras de sentido, esto es, ámbitos de acción que nos permiten hacer frente a esta inmensidad agobiante de sin-sentido. Holzapfel (2016) organiza estas fuentes, por un lado, en fuentes ocasionales y en fuentes persistentes. Las primeras, serían aquellas circunstancias en nuestra vida (un encuentro, la lectura de una página web, una apuesta en un casino) que podrían llegar a transformarse, a la larga, en fuentes más duraderas, y, por tanto, más estables, como fuentes persistentes de sentido. Por otro lado, sugiere la existencia de fuentes programáticas, «que tienen que ver con la proyección de mundo, como la técnica, la tecnología, la ciencia, el derecho, la moral, la religión, la propia filosofía, el arte», y de fuentes referenciales, que "son las que están en la retaguardia, como el "cielo estrellado" del sentido [...] el saber, el sexo-erotismo-amor, el poder...» (Holzapfel, 2016).

Empero, se requiere un tipo distinto de fuentes, a fin de garantizar la semántica del sentido. Holzapfel las denomina fuentes icónicas, «que son las señalizaciones y símbolos del sentido, es decir, sus sustancializaciones» (Villarino, 2008: 72). Como asevera Campos Winter: «Las fuentes icónicas de sentido se muestran como imágenes referidas desde las que brota sentido como puede ser la imagen de un crucifijo, de una bandera, de un escudo de armas o de un viejo barbón, por ejemplo» (CamposWinter, 2017: 17).

Cuando el sentido se retira, surge esta fascinación por estar renovándolo con fuentes ocasionales transformadas en icónicas: «Únicamente nos apoyamos en fuentes nada más que icónicas, que ya dejaron de ser fuentes icónicas naturales [...] son aparatos, máquinas, artefactos» (Holzapfel, 2016). Tales cadenas de sentido, cree el filósofo, proveen una secuencia de sentidos en un orden universal/particular, de manera que lo que se erige es algo parecido a una «torre de sentido», que escalaría hasta un universal que podría interpretarse, finalmente, como el ser mismo o incluso Dios. Este trasfondo que parece designar la ausencia de sentido, trae aparejada en la historia del hombre una determinada concep- 
ción antropológica (el homo romanus christianus o el ser humano cartesiano, por ejemplo), cuya función sería contrarrestar esa posibilidad de sin-sentido. En el caso de Holzapfel (2016), su concepción antropológica revela a un ser humano esencialmente frágil, reflejado en el homo labilis de Ricoeur o en el sujeto débil de Vattimo. En cuanto seres frágiles que somos en esa búsqueda permanente de sentido, requerimos construir por doquier fuentes icónicas que hagan frente a un sin-sentido, que siempre está al acecho. Seres tan frágiles, que Peinado afirmará:

La llamada muerte del sentido (nombre calcado finalmente de la muerte de Dios) procede de la derrota de las fuentes programáticas: no solo se ha cuestionado unas formas históricas concretas (que en los dos últimos siglos han sido sustituidas), sino la misma posibilidad de que una fuente histórica pueda dotar de sentido, dejando a los individuos desguarnecidos (Peinado, 2020: 17).

Paradójicamente, y desde el mundo de la Inteligencia Artificial (IA), hay un particular objeto tecnológico que parece disiparse en el límite entre la idea de una fuente referencial y una fuente icónica de sentido: el videojuego con IA. No cabe duda de que, por sí mismo, el juego, como fenómeno existencial fundamental, forma parte definitiva de la dimensión del sentido. Así lo corroboran autores como Huizinga (2000), Caillois (1958), Fink (1995) y el propio Holzapfel (2005). De este modo, no dudaríamos, con Holzapfel (2005), en ubicarlo como una decisiva fuente referencial de sentido. Sin embargo, no se trata acá del simple juego como fundamento, o si se quiere, como fenómeno existencial. Al contrario, se trata justamente de que la tecnología, por decirlo así, se apropia de este fundamento, y lo pone a disposición - pudiéramos decir con Heidegger (1983), lo impone- de un juego preferentemente agonal. Coincidentemente, la película The Matrix (1999) parece ser un buen ejemplo de lo que digo. Lo que se busca implantar en los videojuegos mediante la IA, en general, es la idea de Agentes Software Inteligentes, en cuanto a que el videojuego no requeriría de la IA si no se tratara, a la larga, precisamente de una acción tipo objetivo/estrategia (Sotillo Mallo y Martínez Ruiz, 2015). Así, y si nos fijamos bien, las nociones de imposición, simulacro y fuentes icónicas/ referenciales de sentido, es decir, los conceptos claves abordados en este trabajo, parecen superponerse en la propia ontología del videojuego con IA, en una deriva tecnológica con alcances ontológicos, éticos y antropológicos insospechados. La posible conexión de este caso de hiperrealidad con la imagen del niño-hombre de Heráclito, jugando, curiosamente, un juego de guerra (Inostroza, 2000: 13), escapa, sin embargo, a los alcances de este escrito.

\section{Conclusiones/Discusión}

La realidad de la hiperinformación, que supone no solo plataformas múltiples de hiperconexión, sino fundamentalmente sentidos y «hermenéuticas» distintas, se ha revelado, paradojalmente, tan convertible como los propios datos que transmite. Parafraseando a Malabou (2011), diríamos que los objetos tecnológicos nunca se presentan como objetos vacíos, sino que siempre suponen una cierta mutación ontológica. Dicha transformación, desde luego, es la de nosotros mismos. A modo sinóptico, el siguiente sería el propio dispositivo «teórico» de nuestros autores: 


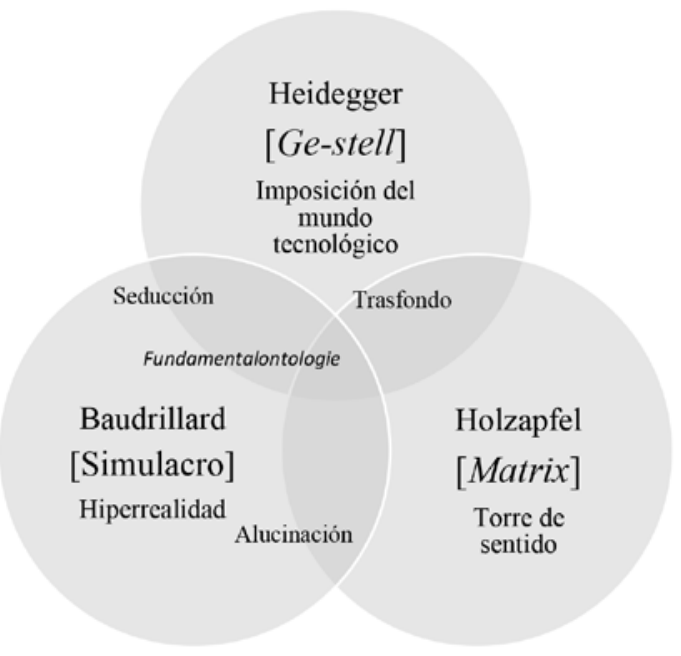

Este marco ontológico - el nuevo Leviatán- es anticipado por Heidegger con su idea del Ge-stell. El Ge-stell es la tecnología (el 6G, la criogenia, el control mental de drones, la web sensorial, mis credenciales ORCID, etc.), pero especialmente nuestra dis-posición ante la técnica. De ahí que, querámoslo o no, caminemos siempre al filo de esta navaja tecnológica. Baudrillard va más lejos todavía. Su interpretación de nuestra era como la era del simulacro, implica una diferencia ontológica radical. El simulacro extingue la diferencia realidad/abstracción, y, por lo mismo, la de realidad/ciencia ficción. No es que lo simulado deje de ser real, sino que esta nueva realidad deja de tener un origen en una determinada productividad, y pasa a convertirse, como lo veíamos en la convertibilidad ontológica del Ge-stell, en hiperrealidad. Para Holzapfel, en cambio, el límite es el trasfondo: el punto en que el sentido queda a un paso del sin-sentido. La zona cero del sentido. Pudiera incluso decirse que aquí yace la verdadera matrix, la que, en pleno vértigo posmoderno, parece ser, sobre todo, una matrix tecnológica. Solo así podemos explicarnos la casi compulsiva generación de fuentes icónicas de sentido. Una visión futurista perfectamente pudiera mostrarnos a una tribu de replicantes, armados - es casi seguro- de una tecnología hoy inimaginable, pero, sin embargo, abocados a esa tarea casi mística de dotación de sentido.

\section{Bibliografía}

BAUDRILLARD, Jean (1980). El intercambio simbólico y la muerte. Caracas: Monte Ávila.

BAUDRILLARD, Jean. (2000). De la seducción. Madrid: Cátedra.

BOSTROM, Nick (2003). «Are you living in a computer simulation?». Philosophical Quarterly (53), 243-

255. Recuperado de https://tinyurl.com/r72twv

CAILLOIS, Roger (1958). Teoría de los juegos. Barcelona: Seix Barral.

CAMPOS WINTER, Hugo (2017). «Sentido del sentido en Víctor Frankl y Cristóbal Holzapfel: Elementos para un metasentido del mundo de la vida». Documentos Lingüísticos y Literarios UACh, (35), 8-24. Recuperado de https://tinyurl.com/yxtyadzy 
CHALMERS, David (2007). «La matrix como metafísica». Discusiones Filosóficas, 8(11), 209-254. Recuperado de https://tinyurl.com/y6erudjr

FEENBERG, Andrew (2005). «Teoría crítica de la tecnología». Revista Iberoamericana de Ciencia, Tecnología y Sociedad, 2(5), 109-123. Recuperado de: https://tinyurl.com/y4sn9mms

FINK, Eugen (1995). Grundphänomene des menschlichen Daseins. Friburgo: Karl Alber.

GUILLÉN, Miguel A., LÓPEZ AYUSO, Belén, PANIAGUA, Enrique y CADENAS, J. Manuel (2015). "Una revisión de la cadena datos-información-conocimiento desde el pragmatismo de Peirce». Documentación de las Ciencias de la Información, 38, 153-177. DOI: 10.5209/rev_DCIN.2015. v38.50814

HAN, Byung-Chul (2014). La sociedad de la transparencia. Barcelona: Herder.

HEIDEGGER, Martin (1983). Ciencia y técnica. Santiago: Universitaria.

HOBBES, Thomas (1980). Leviatán. Madrid: Editora Nacional.

HOLZAPFEL, Cristóbal (2005). A la búsqueda del sentido. Santiago: Sudamericana.

HOLZAPFEL, Cristóbal (2016). Pensando sobre el sentido 3 [Archivo de video], 30 julio. Recuperado de https://tinyurl.com/y59af6j8

HOLZAPFEL, Cristóbal (2018). Nada. Santiago: RIL.

HOLZAPFEL, Cristóbal (2020). Danzando alrededor del sol y preguntándonos sobre el sentido [Archivo de video], 17 agosto. Recuperado de https://tinyurl.com/y2qv62u7

HUIZINGA, Johan (2000). Homo ludens. Madrid: Alianza Editorial.

INOSTROZA, Rodrigo (2000). «Nueva interpretación de Heráclito». Diálogos, (76), 7-28. Recuperado de https://tinyurl.com/yy67ya7o

LEYTON, G. (2018). «Baudrillard. Teoría de la hiperrealidad». Aion.mx.

LINARES, Jorge (2003). «La concepción heideggeriana de la técnica: Destino y peligro para el ser del hombre». Signos Filosóficos, (10), 15-44. Recuperado de https://tinyurl.com/yao3h4v7

LÓPEZ ARAIZA BRAVO, Hugo (2012). "Cómo y por qué una Filosofía de la tecnología». Argumentos de Razón Técnica, (15), 11-124. Recuperado de https://tinyurl.com/y56h8r3m

LÓPEZ DEVESA, Emilio Juan (2001). «¿Tecnología y ciencia, o sólo tecnología? Hacia una comprensión de las relaciones ciencia-tecnología». Argumentos de Razón Técnica, (4), 195-218. Recuperado de https://tinyurl.com/y6kalyvv

LUNA ALCOBA, Manuel (2013). Ensayos de Tecnodicea sobre la bondad de la ciencia, la libertad del mercado y el origen de la máquina. Google Libros.

MALABOU, Catherine (2011). The Heidegger change: On the fantastic in philosophy. New York: Suny Press.

NAVARRO CORDÓN, Juan Manuel (1966). «Sentido de la ontología fundamental en Heidegger». Logos. Anales del Seminario de Metafísica, 1, 29-51. Recuperado de https://tinyurl.com/y22m22qx

PEINADO, Carlos (2020). "Sentido y sinsentido en la poesía española reciente: consecuencias en los vectores simbolista y antisimbolista». En Nieto, Miguel (coord.). El escritorio y el mundo: hallazgos de sentido para la creación literaria. Madrid: Verbum, págs. 15-58.

QUINTANILLA, Miguel Ángel (2005) Tecnología: un enfoque filosófico y otros ensayos de filosofía de la tecnología. México: Fondo de Cultura Económica.

RAMOS, Sergio (2019). «Masacre en Nueva Zelanda fue transmitida en Facebook por el atacante». Social Geek, 15 marzo. Recuperado de https://tinyurl.com/y5c5bzc9

SALOMÓN AMAYA, Ulises (2015). «La esencia de la Técnica (Das Ge-stell) como un habitar (Whonen)». Teoría y Praxis, (27), 49-68. DOI: 10.5377/typ.v0i27.2849 
SOTILLO MALLO, Javier y MARTÍNEZ RUIZ, Cristian (2015). «Inteligencia artificial en los videojuegos». Recuperado de https://tinyurl.com/y3zffkvz

VASKES SANTCHES, Irina (2008). «La transestética de Baudrillard: simulacro y arte en la época de simulación total». Estudios de Filosofía, (38), 197-219. Recuperado de https://tinyurl.com/y6j76uhj VILLARINO, Hernán (2008). «Sentido, sin-sentido y realidad en Frankl, Holzapfel y Zubiri». GPU, 4(1), 69-77. 